BULLETIN (New Series) OF THE

AMERICAN MATHEMATICAL SOCIETY

Volume 46, Number 4, October 2009, Pages 667-668

S 0273-0979(09)01270-1

Article electronically published on June 30, 2009

\title{
ON A. WEIL
}

\author{
YUTAKA TANIYAMA, \\ WITH TRANSLATION AND INTRODUCTION BY \\ MARK GORESKY AND KEIKO KAWAMURO
}

\section{INTRODUCTION}

When he was a college student, Taniyama studied the papers of A. Weil. He was apparently so impressed by this work that in 1953 he wrote a small note, "On Weil", which was published in Sugaku no ayumi, volume 1, no. 1, the periodical of the New Mathematical Society, of which Taniyama was one of the original founders. It is reprinted in Taniyama's collected works, in the original Japanese. It was not until 1955 that Taniyama met Weil in person, at the International Symposium on Number Theory, held in Tokyo.

In his note, Taniyama expresses almost contradictory opinions, at one point praising Weil for his insight, creativity, and technical power, but at the same time criticizing Weil for not going far enough. In the penultimate paragraph Taniyama asks if there is any room left for revolutionary ideas in mathematics. The article ends with a devastating opinion concerning the state of Japanese mathematics of the 1950s.

\section{ON A. WEIL}

Andre Weil is perhaps the best in the world, among active mathematicians, except for C. L. Siegel. [Weil is] a professor at the University of Chicago. He is outspoken. His criticism is harsh. His unbiased frankness, along with his broad viewpoint and deep insight, is one of the driving forces for the Bourbaki movement. But his reputation suffers, even among the less vocal senior mathematicians. However, mathematics would suffocate, were it not for its open attitude, which does not immediately dismiss the Bourbaki movement.

One can see his deep insight and broad viewpoint, for example, in his essay, "L'avenir des Mathematiques" or his lecture at the International Congress in 1950. Since he makes huge leaps and speculates fearlessly, it is sometimes hard for us to trust him. However, it is dangerous for mediocre people like us, to judge the thoughts of a genius.

It is well known that Bourbaki was formed by people around Weil, to counter the entrenched tradition of analysis in France. But we should not overlook their deep knowledge of classical mathematics, which lies beneath a surface of modernism. At one time, mathematics was regarded as pure abstraction, a set of axioms, a theory of noncontradiction. Awaking in the 1930's from this fascinating dream, "modern"

Received by the editors May 29, 2009.

(C)2009 American Mathematical Society Reverts to public domain 28 years from publication 
mathematics found this formalism quite unsatisfactory. What can have "meaning" in an abstract, noncontradictory theory?

In order to be meaningful, it [mathematics] must be able to abstract, integrate, and reconstruct classical results into broad perspectives. It has been said that the goals of modern mathematics are reconstruction and development. Moreover, a subject that limits itself to a single abstract foundation is exposed to the danger of becoming purely academic. (We have already observed these signs in some mathematical communities in America.) We should note that the insight cultivated by the tradition of the Bourbaki circle avoids such dangers.

In any case, Weil is, in this sense, a representative of modern mathematics. Therein lies his secret, but also his end.

Though his thoughts are clear, his papers are tersely written with a variety of approaches and technical methods, and they are quite difficult to read. Let us choose the most interesting three examples from his papers: the theory of indeterminate [Diophantine] equations, the three sequels about algebraic geometry, and the theory of class fields and $L$-functions. Here we notice several common remarkable features. What should be abstracted and generalized? This is the first problem. For this, Weil accurately extracts an essential property or a key idea, from a classical theory. The next problem is to carry out this scheme. Along the way, of course, he encounters many significant obstructions. At this point, most mathematicians would give up or seek a detour. But Weil never changes his original scheme. He conquers the obstructions one by one. His next most important talents are his strength and persistence. They are responsible for the depth of his achievements, which go beyond simple abstractions.

However, a talented person is attracted to diverse areas. Since he attacks too many problems, he has a tendency to insufficiently investigate a single problem. This is why his important results are lacking in delicacy.

Moreover, there is one further question. Indeed, they [Bourbaki] have established solid foundations for mathematics, and they have developed vast generalizations. But so what? This is an issue for modern mathematics itself. Should we always rely on the 19th century [for our source of problems]? A completely different area, an unexpected development, a deep relation between several branches which is more than just a formal analogy, do such things no longer exist? It is not possible to pioneer a new such world via Weil's approach. But it may become possible, if a genius of the century happens along. Perhaps it is irrelevant for us to dream about undiscovered worlds, since so many unsolved problems are left at the present time, even in the framework of modern mathematics. Rather, we should probably wait for the second and the third Weil.

Earlier, I mentioned Weil's strength. Siegel, who is far more creative than Weil, also surpasses Weil in terms of strength. For the many mathematicians of our country, who love abstract formalism but lack strength, to strive for a depth of creativity would certainly hit them at their weak point!

Department of Mathematics, University of Tokyo

School of Mathematics, Institute for Advanced Study. Princeton, New Jersey

School of Mathematics, Institute for Advanced Study; And Department of MatheMATICS, UNIVERSITY OF IOWA 